\title{
Meta-learning Haptic Exploration of Simple 3D Objects
}

\author{
Alexandra Moringen ${ }^{1}$, Sascha Fleer and Helge Ritter ${ }^{1}$
}

\begin{abstract}
The vision of this work is to build a generalized model of haptic exploratory procedures that can be employed by a wide range of robotic platforms in different environments. Main challenge of combining deep learning and robotics is currently the time-efficiency of sample acquisition. Having to learn to perform a sequence of interactions with the environment, in which next action may depend on the previously acquired data, adds an extra complexity to the task of quickly teaching a robot to perform a haptic task. A meta-model for exploratory procedures built with data acquired by multiple robot platforms in simulation and online, that can be quickly adapted to a new robot or task, may offer a solution to this challenge. Here, we describe our approach to meta-learning of a haptic exploration policy offline, and demonstrate preliminary results achieved after only two gradient steps performed online with data acquired by a robot. We exemplify our approach on the task of learning to classify four simple three-dimensional objects. The meta-learner's learning rule enables us to learn a generalized policy representing experience from different types of data available offline, and quickly adapt it to a new task - online classification of objects based on a sequence of tactile sensor measurements acquired by a robot. Tactile measurements are acquired through performing of three haptic glances per object at poses generated by the trained closedloop control policy. According to the principles of interactive perception, we optimize the closed-loop location control policy, integration of the data from the past iterations, and a classifier that classifies the objects all in one go. We employ the model resulting from a meta-training to control haptic exploration on the real robot. The classification accuracy achieved after two gradient updates of the meta-model performed with the data acquired by the robot is $93.7 \%$ evaluated on a batch of 16 samples.
\end{abstract}

\section{INTRODUCTION AND METHOD OVERVIEW}

The focus of this work is on design of a cognitive architecture that addresses the main challenges of learning haptic exploration, and in particular with a robot: integrating previous experience, optimizing exploration sequences, adapting to new types of sensors, while still using available but datainefficient optimization techniques such as reinforcement learning that are difficult to apply on a robot. With the meta-learning approach [1] applied to previously developed HAM [2], our long-term goal is to learn a haptic attention controller that generalizes over different types of tactile sensor measurements acquired mainly in simulation, e.g. employed in the shadow fingers, or in the flat sensor grids, such as Myrmex. In this work, we show how to accumulate experience from the training based on small set of manually acquired real data without using the robot (rig data set) and a large simulated haptic data (see Figure 2). The goal is to learn a policy that performs an optimal sequence of haptic glances, primitive haptic actions that in the current

\footnotetext{
${ }^{1}$ Center for Cognitive Interaction Technology, Bielefeld University
}

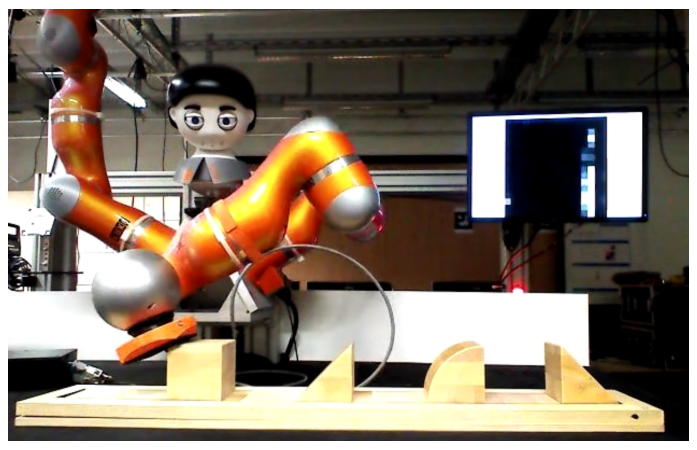

Fig. 1. Target setup performing haptic classification task of four displayed objects. The setup consists of Kuka robot-arm with Myrmex sensor array attached to it. Target task consists of learning to classify four objects by performing an optimal sequence of three haptic glances per object. An example of glance data corresponding to the pose of the robot arm is illustrated in the monitor in the right upper corner of the picture. For a detailed robot setup and HAM description, we refer to [2], [3]

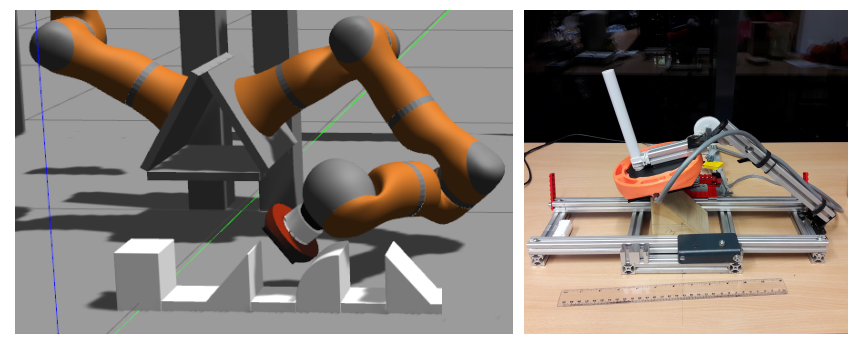

Fig. 2. Data acquired for meta-training. Left: physics-driven simulation environment Gazebo with the four objects, Kuka robot-arm and a Myrmex sensor array producing simulated tactile data. Right: rig with a Myrmex sensor for manual acquisition of real sensor data offline.

experiment we parameterize by a one-dimensional rotation and translation of the end-effector approaching the object from above ${ }^{1}$. We refer for further information to the following link http://techfak.uni-bielefeld.de/ persons/abarch/whc_2021.pdf.

\section{REFERENCES}

[1] Chelsea Finn, Pieter Abbeel, and Sergey Levine. Model-agnostic metalearning for fast adaptation of deep networks, 2017.

[2] Sascha Fleer, Alexandra Moringen, Rl. Klatzky, and Helge Ritter Learning efficient haptic shape exploration with a rigid tactile sensor array. btxhak.pdf, January 2020.

[3] Alexandra Moringen, Sascha Fleer, Guillaume Walck, and Helge Ritter Attention-based robot learning of haptic interaction. In Ilana Nisky, Jess Hartcher-O'Brien, Michaël Wiertlewski, and Jeroen Smeets, editors, Haptics: Science, Technology, Applications, pages 462-470, Cham, 2020. Springer International Publishing.

\footnotetext{
${ }^{1}$ An introductory video to the functionality of HAM and haptic glances is available here: https: / / link. springer.com/chapter/ $10.1007 / 978-3-030-58147-3 \_51$
} 